\title{
Stochastic Modeling and Quality Evaluation of Workflow Systems Based on QWF-Nets*
}

\author{
Yunni Xia, Hanpin Wang, Chunxiang Xu, and Liang Li \\ School of Electronics Engineering and Computer Science \\ Peking University, China \\ \{xiayunni, whpxhy\}@pku.edu.cn
}

\begin{abstract}
Quality (QOS) prediction is one of the most important research topics of workflow management system. In this paper, we propose the SWQ approach to analytically evaluate QOS of workflow systems based on QWF-net, which extends traditional WF-net by associating tasks with exponential response time and exponential TTF (time-tofailure). The comparison between simulative and analytical results in the case study indicates that the SWQ approach achieves satisfactory accuracy. The paper concludes with a comparison between the SWQ approach and other related work.
\end{abstract}

\section{Introduction}

With the advent and evolution of global scale economies, organizations need to be more competitive, efficient and flexible. In the past decade, workflow techniques have been widely used to address these needs.

However, among many research topics of workflow, performance/quality analysis is yet to be given the importance it deserves. Techniques and models [1-8] for QOS evaluation are still preliminary and limited. This paper introduces an analytical approach, the SWQ (meaning a stochastic approach for quality evaluation of workflow systems) to address the need for QOS evaluation. The SWQ approach is based on QWF-net (stochastic WF-net) model, which is an extension of traditional WF-net where tasks are associated with exponential response time and exponential TTF (time-to-failure). By mapping the execution of QWF-net into a homogeneous continuous Markovian process, the SWQ approach calculates its expected completion-time and reliability.

Through comparing simulative results and results obtained by SWQ approach, the case-study indicates that our approach achieves satisfactory accuracy. The paper concludes with a comparison between the SWQ approach and related work.

\footnotetext{
* Supported by the National Natural Science Foundation of China under Grant No. 60173002 and the National Grand Fundamental Research 973 Program of China under Grant No. 2002CB312004.
} 


\section{QWF-Net for Quality Prediction}

The Workflow net (WF-net) proposed by van der Aalst is a high level Petri Nets with two special places $i$ and $o$, which indicate the beginning and the end of the modeled process. There exist four routing patterns in WF-nets, namely sequence, parallel, selective and iterative. WF-net does not care the concept of time, but sometimes we need to consider time aspect in workflow management systems. For example, we want to know the completion time of the whole workflow net or of some subnets so that we can decide whether the arrangement of the workflow system meets constraint of time. So introducing time concept into WF-net is necessary.

This section extends WF-net to QWF-net by associating an exponential response time and exponential TTF (Time-to-failure) with each task.

Definition 1. (QWF-net) $N=(P, T, T a s k, \lambda, \mu)$ is a QWF-net if and only if:

1. $N$ is structurally a WF-net

2. SPLIT/JOIN transitions (black thin bars in Fig.1) fire immediately and have response time of 0

3. SPLIT/JOIN transitions never fail

4. The set Task $\subseteq T$ denotes the set of transitions excluding SPLIT/JOIN transitions (as illustrated by white bars in Fig.1)

5. Each task has an exponential response time. A function $\lambda:$ Task $\rightarrow$ Real is used to identify parameter (also known as execution-rate) of the exponential response time of each task

6. Each task fails independently and has an exponential TTF. A function $\mu$ : Task $\rightarrow$ Real is used to identify parameter (also known as failure-rate) of the exponential TTF of each task

It easily follows that QWF-net is identical with WF-net in construction aspect. Therefore, structural properties of WF-net also follow in QWF-net: there should be no dead tasks; the procedure should terminate eventually; at the moment the procedure terminates there should be one token in sink place o and all the other places are empty; the definition of reachable markings and its corresponding calculation methods for WF-net can also be applied to QWF-net.

\section{Quality Evaluation Based on QWF-Net}

Besides assumptions about exponential task response time and TTF, this paper also assumes that:

1. The control flow randomly chooses its path on XOR-split according to given choice probabilities. For generality, this paper uses a function se : Task $\rightarrow$ Real to denote the probability that each task is selected when its preceding task finishes execution. Note that, if a task is not on any XOR-split, its choice probability equals 1 , otherwise smaller than 1 
2. The control flow skips loop when current iteration finishes according to a given probability. For generality, this paper uses a function $l o:$ Task $\rightarrow$ Real to denote the probability that the control flow skips each task when current iteration finishes. Note that, if a task is not on any loop, its corresponding skipping probability equals 1 , otherwise smaller than 1 . Therefore, for a task $t_{i}$, its expected number of loop iterations is $\frac{1}{l o\left(t_{i}\right)}$, following a geometric distribution.

\subsection{Evaluating the Expected Completion Time (ECT)}

This subsection presents an analytical method to calculate the expected completion time of QWF-net based on response time of each task. Let $U(t)$ denote the set of operational tasks in QWF-net at time $t$ (execution begins at time 0 ), then its state-space (denoted by $S$ ) is obtained through mapping each reachable marking into a corresponding set of operational tasks.

For any reachable marking $M_{1} \in\left[M_{0}>\right.$ where no SPLIT/JOIN transitions are activated, there exists a state which records all operational tasks in this marking. Since SPLIT/JOIN transitions fire immediately with response time 0, there is no need to generate states indicating whether SPLIT/JOIN transitions are activated or not. Take Fig.1 for example, the marking illustrated in this figure where $P_{1}$ contains a token is one such negligible marking since this marking merely indicates that $A N D-$ split $_{1}$ transition is activated. Also note that the marking where only sink place contains a token is mapped into an absorbing state which records no task is operational, meaning all tasks are idle and the control flow terminates.

The state space of $U(t)$ of Fig.1 are illustrated in Table.1. Note that, there exist more than one initial-state since $X O R-S P L I T_{1}$ may generate one token into place $P_{4}, P_{5}$ or $P_{6} . S_{12}$ is the absorbing state.

Table 1. State space

\begin{tabular}{cc|cc}
\hline state & operational tasks & state & operational tasks \\
\hline$S_{1}$ (Initial-state) & $\left\{t_{1}, t_{6}\right\}$ & $S_{7}$ & $\left\{t_{3}, t_{8}\right\}$ \\
$S_{2}$ (Initial-state) & $\left\{t_{1}, t_{7}\right\}$ & $S_{8}$ & $\left\{t_{3}\right\}$ \\
$S_{3}$ (Initial-state) & $\left\{t_{1}, t_{8}\right\}$ & $S_{9}$ & $\left\{t_{6}\right\}$ \\
$S_{4}$ & $\left\{t_{1}\right\}$ & $S_{10}$ & $\left\{t_{7}\right\}$ \\
$S_{5}$ & $\left\{t_{3}, t_{6}\right\}$ & $S_{11}$ & $\left\{t_{8}\right\}$ \\
$S_{6}$ & $\left\{t_{3}, t_{7}\right\}$ & $S_{12}$ (Absorbing-state) & $\varnothing$ \\
\hline
\end{tabular}

As mentioned in the assumption, a task $t_{i}$ has exponential response time with parameter $\lambda\left(t_{i}\right)$ and the probability that control flow skips task $t_{i}$ when current iteration of $t_{i}$ finishes is $l o\left(t_{i}\right)\left(l o\left(t_{i}\right)=1\right.$ if $t_{i}$ is not on loop). The number of loop iterations of $t_{i}, N_{t_{i}}$, is a geometric distributed random variable with parameter $l o\left(t_{i}\right)$. Since task may be iteratively executed, this paper uses $D_{t_{i}}$ to denote the total response time of $t_{i}$ considering iterative execution and $X_{t_{i}}$ to denote 


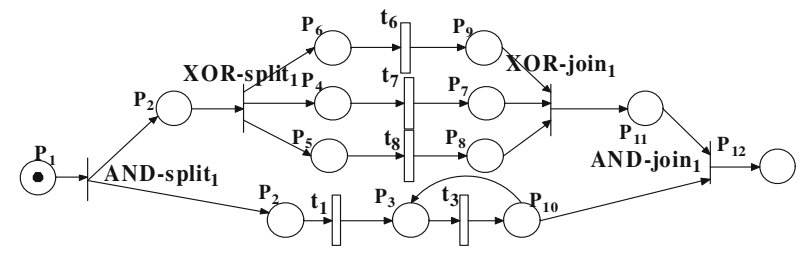

Fig. 1. A QWF-net example (Case $)_{5}$

response time of one single iteration. According to the definition of QWF-net, $X_{t_{i}}$ follows exponential distribution. Therefore, the cumulative-distribution-function $(\mathrm{CDF})$ of $D_{t_{i}}$ is given as

$$
\begin{aligned}
F(y) & =\operatorname{Prob}\left\{D_{t_{i}} \leq y\right\} \\
& =\sum_{K=1}^{\infty} \operatorname{Prob}\left\{N_{t_{i}}=k\right\} \operatorname{Prob}\left\{N_{t_{i}} \times X_{t_{i}} \leq y \mid N_{t_{i}}=K\right\} \\
& =\sum_{K=1}^{\infty} \operatorname{lo}\left(t_{i}\right)\left(1-l o\left(t_{i}\right)\right)^{K-1} E_{K}(y)
\end{aligned}
$$

where $E_{K}(y)$ denotes the CDF of $\mathbf{K}$-phase Erlang distribution.

Then, the density-distribution-function (PDF) of $D_{t_{i}}$ is given as

$$
\begin{aligned}
f(y) & =F^{\prime}(y) \\
& =\sum_{K=1}^{\infty} l o\left(t_{i}\right)\left(1-l o\left(t_{i}\right)\right)^{K-1} \frac{\lambda\left(t_{i}\right)\left(y \lambda\left(t_{i}\right)\right)^{K-1}}{(K-1) !} e^{-\lambda\left(t_{i}\right) y} \\
& =\lambda\left(t_{i}\right) \operatorname{lo}\left(t_{i}\right) e^{-y \lambda\left(t_{i}\right)} \sum_{K=1}^{\infty} \frac{\left(\left(1-l o\left(t_{i}\right)\right) y \lambda\left(t_{i}\right)\right)^{K-1}}{(K-1) !} \\
& =\lambda\left(t_{i}\right) \operatorname{lo}\left(t_{i}\right) e^{-y \lambda\left(t_{i}\right)} \times e^{\left(1-l o\left(t_{i}\right)\right) \lambda\left(t_{i}\right) y} \\
& =\lambda\left(t_{i}\right) \operatorname{lo}\left(t_{i}\right) e^{-\lambda\left(t_{i}\right) l o\left(t_{i}\right) y}
\end{aligned}
$$

where $\frac{\lambda\left(t_{i}\right)\left(y \lambda\left(t_{i}\right)\right)^{K-1}}{(K-1) !} e^{-\lambda\left(t_{i}\right) y}$ is the PDF of the K-phase Erlang distribution.

According to the equation above, $D_{t_{i}}$ follows exponential distribution with parameter $\lambda\left(t_{i}\right) l o\left(t_{i}\right)$. Since the total response time $D_{t_{i}}$ of every task $t_{i}$ follows exponential distribution, $U(t)$ is a homogeneous continuous Markovian process. The infinitesimal generator matrix $Q$ of $U(t)$ is given as

$$
q_{i, j}= \begin{cases}l o\left(t_{l}\right) \times \lambda\left(t_{l}\right) \times \prod_{t_{m} \in N E W(i, j)} s e\left(t_{m}\right) & \text { if } S_{i} \underset{t_{l}}{\rightarrow} S_{j} \\ -\sum_{1 \leq r \leq W, r \neq i} q_{i, r} & \text { if } i=j \\ 0 & \text { else }\end{cases}
$$

where $l o\left(t_{l}\right) \times \lambda\left(t_{l}\right)$ denotes the parameter of the exponential random variable $D_{t_{l}}, W$ denotes the number of states in the state space, and $q_{i, j}$ denotes the transition rate from state $S_{i}$ to $S_{j}$. 
Relation $S_{i} \underset{t_{l}}{\rightarrow} S_{j}$ means that $S_{j}$ is the resulting state of $S_{i}$ if the operational task $t_{l}$ in $S_{i}$ finishes execution and becomes idle. Note that, there may exist more than one resulting states of $S_{i}$ when $t_{l}$ becomes idle because transition may activate choice (XOR-split). Those resulting states are viewed as different types in the Markovian chain according to the phase-type property and $\prod_{t_{m} \in N E W(i, j)} s e\left(t_{m}\right)$ denotes the occurrence probability of $S_{j}$ among all types, where $\boldsymbol{N} \boldsymbol{E} \boldsymbol{W}(\boldsymbol{i}, \boldsymbol{j})$ denotes the set of newly-emerging operational tasks in the transition from state $S_{i}$ to $S_{j}$.

Take the transition from state $S_{8}$ to $S_{12}$ for example, we have its transition rate $q_{8,12}$ as $l o\left(t_{3}\right) \lambda\left(t_{3}\right)$ according to Eq.3.

The expected time which a state $S_{i}$ takes to reach the absorbing state (timeto-termination) is defined as $E T T_{S_{i}}$, where

$$
\begin{aligned}
& \operatorname{ETT}_{S_{i}}= \begin{cases}0 & \text { absorbing-state } \\
\frac{1}{E_{S_{i}}}+\sum_{1 \leq k \leq W, k \neq i} \frac{q_{i, k} \times E T T_{S_{k}}}{E_{S_{i}}} & \text { else }\end{cases} \\
& \text { where } E_{S_{i}}=\sum_{1 \leq j \leq W, j \neq i} q_{i, j}
\end{aligned}
$$

According to the equation above, the expected time for $S_{i}$ to reach termination is the expected duration of state $S_{i}\left(\frac{1}{E_{S_{i}}}\right)$ plus the averaged (by occurrence probabilities) time-to-termination of its immediate succeeding states.

Therefore, the expected completion-time of QWF-net is the averaged(by occurrence probability) time-to-termination of all initial states. Let Init and $B U S Y_{S_{i}}$ denote the set of initial states and the set of operational tasks in state $S_{i}$ respectively, then the QWF-net's expected completion time ECT is

$$
E C T=\sum_{S_{i} \in \text { Init }}\left(E T T_{S_{i}} \times \prod_{t_{j} \in B U S Y_{S_{i}}} s e\left(t_{j}\right)\right)
$$

\subsection{Evaluating Reliability}

The reliability estimate of $t_{i}$ is obtained through integrating the probability that its TTF is larger than $t$ (meaning the probability that $t_{i}$ does not fail till time $t$ ) multiplied by PDF of $D_{t_{i}}$ over the interval from 0 to $\infty$. Therefore, reliability of task $t_{i}, R_{t_{i}}$, is given by

$$
\begin{aligned}
R_{t_{i}} & =\int_{0}^{\infty} \lambda\left(t_{i}\right) \operatorname{lo}\left(t_{i}\right) e^{-\lambda\left(t_{i}\right) \operatorname{lo}\left(t_{i}\right) t} \times \operatorname{Prob}\left\{T T F_{t_{i}}>t\right\} d t \\
& =\int_{0}^{\infty} \lambda\left(t_{i}\right) \operatorname{lo}\left(t_{i}\right) e^{-\lambda\left(t_{i}\right) \operatorname{lo}\left(t_{i}\right) t} \times e^{-\mu\left(t_{i}\right) t} d t \\
& =\frac{\lambda\left(t_{i}\right) \operatorname{lo}\left(t_{i}\right) \int_{0}^{\infty}\left(\lambda\left(t_{i}\right) \operatorname{lo}\left(t_{i}\right)+\mu\left(t_{i}\right)\right) e^{-\left(\lambda\left(t_{i}\right) l o\left(t_{i}\right)+\mu\left(t_{i}\right)\right) t} d t}{\lambda\left(t_{i}\right) \operatorname{lo}\left(t_{i}\right)+\mu\left(t_{i}\right)} \\
& =\frac{\lambda\left(t_{i}\right) \operatorname{lo}\left(t_{i}\right)}{\lambda\left(t_{i}\right) \operatorname{lo}\left(t_{i}\right)+\mu\left(t_{i}\right)}
\end{aligned}
$$

where $\lambda\left(t_{i}\right) l o\left(t_{i}\right) e^{-\lambda\left(t_{i}\right) l o\left(t_{i}\right) t}$ is $\mathrm{PDF}$ of the $D_{t_{i}}$ and $e^{-\mu\left(t_{i}\right) t}$ is the probability that TTF (time-to-failure) of $t_{i}$ is greater than $t$. 
Therefore, QWF-net's reliability is the averaged reliability of all initial states

$$
\text { Reliability }=\sum_{S_{i} \in \text { Init }}\left(R_{S_{i}} \times \prod_{t_{j} \in B U S Y_{S_{i}}} s e\left(t_{j}\right)\right)
$$

where $R_{S_{i}}$ denotes the reliability of state $S_{i}$

$$
R_{S_{i}}=\left\{\begin{array}{cl}
1 & \text { absorbing-state } \\
\sum_{\text {Every } S_{j}} \text { satisfying } S_{i} \stackrel{t_{l} S_{j}}{\longrightarrow} R_{t_{l}} \times R_{S_{j}} \times \frac{q_{i j}}{E_{S_{i}}} & \text { else } \\
\text { where } E_{S_{i}} \stackrel{=}{=} \sum_{1 \leq j \leq W, j \neq i} q_{i, j} &
\end{array}\right.
$$

\section{Case Study and Simulation}

This section applies the SWQ approach to some examples and studies its accuracy through a comparison with Monte-Carlo simulation. The examples are given by Fig.2. Tasks involved are listed in Table.2. Case $_{1-4}$ are four simple examples dealing with sequential, parallel, selective and iterative routing modes, respectively. Case $_{5}$ (given earlier in Fig.1) and Case $_{6}$ are more complex examples featured by all routing modes.

A simulation procedure is developed to evaluate expected completion time and reliability of QWF-net. The simulation procedure is similar to simulation

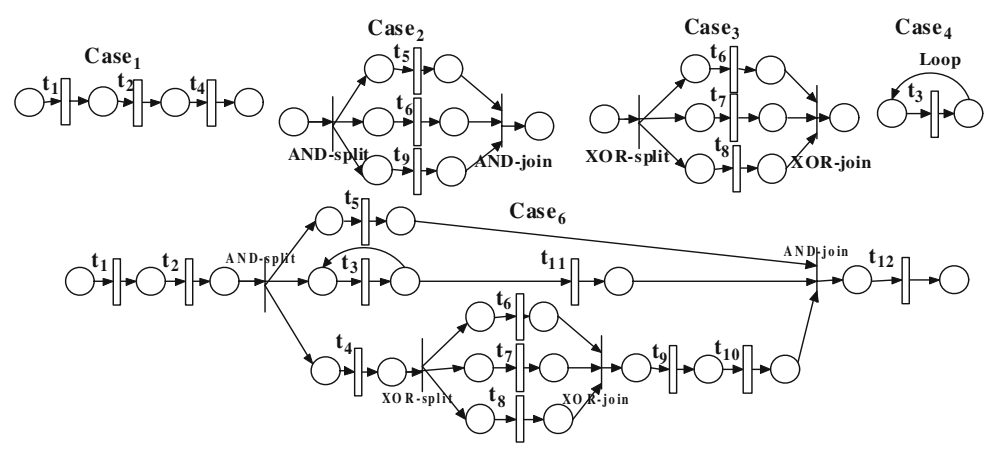

Fig. 2. Cases

Table 2. Tasks involved in the cases

\begin{tabular}{ccccc|ccccc}
\hline Tasks & $\lambda$ & $\mu$ & se & lo & Tasks & $\lambda$ & $\mu$ & se & lo \\
\hline$t_{1}$ & 0.3 & 0.0026 & 1 & 1 & $t_{7}$ & 0.3 & 0.0013 & 0.2 & 1 \\
$t_{2}$ & 0.2 & 0.0028 & 1 & 1 & $t_{8}$ & 0.45 & 0.0014 & 0.1 & 1 \\
$t_{3}$ & 0.4 & 0.0053 & 1 & 0.33 & $t_{9}$ & 0.3 & 0.0064 & 1 & 1 \\
$t_{4}$ & 0.65 & 0.0041 & 1 & 1 & $t_{10}$ & 0.45 & 0.0054 & 1 & 1 \\
$t_{5}$ & 0.6 & 0.0051 & 1 & 1 & $t_{11}$ & 0.8 & 0.0023 & 1 & 1 \\
$t_{6}$ & 0.2 & 0.0037 & 0.7 & 1 & $t_{12}$ & 0.25 & 0.0058 & 1 & 1 \\
\hline
\end{tabular}


algorithms for SPN (stochastic Petri-net) performance analysis, which use random variable generators to decide firing delay of each transition at each simulation run. The procedure is executed for sufficiently many times. At each run, the procedure randomly chooses a path along XOR-split and decides the numbers of loop iterations of loops according to predefined probabilities. Then the procedure uses exponential-distribution random variable generators to generate response time of each task. Also, the program uses random variable generators to generate the TTF of every task. At each run, if all tasks' TTF are greater than their response time (meaning no failure happens during the execution of this task), a success is recorded. The simulative estimates of completion-time is obtained through averaging completion-time of all runs. The simulative estimate of reliability is the ratio of successes to the number of all runs.

Results obtained by simulation (illustrated in normal style) are compared with those by the SWQ approach (illustrated in bold style ) in Table.3. As shown, analytical results is pretty close to simulative results. It indicates that SWQ approach achieves satisfactory accuracy.

Table 3. Comparison between simulative and SWQ results

\begin{tabular}{ccc|lcc}
\hline case & ECT & Reliability & case & ECT & Reliability \\
\hline Case $_{1}$ & $9.8879 / \mathbf{9 . 8 7 1 8}$ & $97.25 \% / \mathbf{9 7 . 1 6 \%}$ & Case $_{4}$ & $7.4942 / \mathbf{7 . 5 0 0 0}$ & $96.07 \% / \mathbf{9 6 . 1 3 \%}$ \\
Case $_{2}$ & $6.5456 / \mathbf{6 . 5 4 8 0}$ & $95.42 \% / \mathbf{9 5 . 3 2 \%}$ & Case $_{5}$ & $11.8686 / \mathbf{1 1 . 8 6 6 1}$ & $93.94 \% / \mathbf{9 3 . 9 9 \%}$ \\
Case $_{3}$ & $4.3883 / \mathbf{4 . 3 8 8 9}$ & $98.60 \% / \mathbf{9 8 . 6 1 \%}$ & Case $_{6}$ & $25.5926 / \mathbf{2 5 . 5 5 3 9}$ & $86.07 \% / \mathbf{8 6 . 1 1 \%}$ \\
\hline
\end{tabular}

\section{Comparison with Related Work}

Research of $[2,3]$ uses a reduction technique to simplify sequential, parallel, selective and iterative routing patterns into a single transition with equivalent QOS estimate, however these methods are not very realistic since they assume tasks in WF-net have deterministic response time rather than nondeterministic as this paper does.

Methods of $[1,7,8]$ are similar to the SWQ approach in that they also model the control flow as continuous Markov chains. These models map execution of each task into each state of CTMC chain and analytically evaluate transition probability or transition rate between states. However these methods can not model parallel execution of more than one tasks in their Markov chains.

Method proposed by [5] develops a simplification technique to simplify four basic routing patterns into a single task with approximate equivalent performance and assumes that the equivalent task still follows exponential distribution if all tasks have exponential response time. However, its assumption that the simplified construct still follows exponential distribution is obviously inaccurate and unrealistic. For instance, $n$ tasks with exponential response time of the same execution rate arranged by sequential routing pattern should be simplified into a single equivalent task of $n$-phase Erlang response time (which is obviously not exponential). 


\section{Conclusion}

This paper proposes the SWQ method to analytically evaluate QOS (expectedcompletion-time and reliability) of workflow systems based on the QWF-net model, which extends traditional WF-net by associating tasks with exponential response time and TTF. This paper also develops a simulation procedure to calculate the simulative QOS results. The comparison between simulative and analytical results in the case study indicates that SWQ approach achieves satisfactory accuracy.

\section{References}

1. J.Klingemann, J.Waesch and K.Aberer. Deriving Service Models in CrossOrganizationalWorkflows. In Proceedings of Int'l Workshop on Reasearch Issues in Data Engineering (RIDE), pages 100-107, 1999

2. Jorge Cardoso, Amit Sheth, John Miller, Jonathan Arnold and Krys Kochut. Quality of service for workflows and web service processes. In Elsevier Transaction on web semantics, 1(3), pages 281-308, 2004

3. Zhangxi Tan, Chuang Lin, Hao Yin, Ye Hong and Guangxi Zhu. Approximate Performance Analysis of Web Services Flow Using Stochastic Petri Net. In Proceedings of Grid and Cooperative Computing GCC 2004 Third International Conference, pages 193-200, 2004

4. Michael C.Jaeger, Gregor Rojec-Goldmann and Gero Muhl. QoS Aggregation in Web Service Compositions. In Proceedings of IEEE International Conference on e-Technology, e-Commerce and e-Service EEE-05, pages 181-185, 2005

5. LIN Chuang, QU Yang, REN Fengyuan and Dan C.Marinescu. Performance Equivalent Analysis of Workflow Systems Based on Stochastic Petri Net Models. In Proceedings of 1st International Conference on Engineering and Deployment of Cooperative Information Systems, pages 64-79, 2002

6. JianQiang Li, YuShun Fan, and MengChu Zhou. Performance Modeling and Analysis of Workflow. In IEEE transaction on SYSTEMS, MAN, AND CYBERNETICSPART A: SYSTEMS AND HUMANS, 34(2), pages 229-242, 2004

7. Michael Gillmann, Jeanine Weissenfels, Gerhard Weikum and Achim Kraiss. Performance and Availability Assessment for the Configuration of Distributed Workflow Management Systems. In Proceedings of 7th International Conference on Extending Database Technology, pages 183-201, 2000

8. Michael Gillmann, Gerhard Weikum and Wolfgang Wonner. Workflow Management with Service Quality Guarantees. In Proceedings of ACM SIGMOD International Conference on Management of Data, pages 228-239, 2002 